\title{
Hacia las unidades de bibliometría en las universidades: modelo y funciones
}

\author{
Daniel Torres-Salinas* y Evaristo Jiménez-Contreras ${ }^{\star *}$
}

Resumen: En los últimos años la bibliometría evaluativa se han consolidado firmemente como herramienta en la gestión de la investigación y toma de decisiones en el ámbito de la política científica. Una de las instituciones donde más está creciendo este uso es en las universidades españolas que cada vez se encuentran en un entorno más competitivo y tienen una mayor necesidad de conocer el rendimiento de sus investigadores. Teniendo en cuenta este contexto en el presente trabajo se expone una propuesta para la creación de unidades de bibliometría en las universidades españolas. El objetivo específico es, por tanto, ilustrar el modelo y funciones de dichas unidades que han de basarse en tres pilares fundamentales: control de las fuentes de la información, realización de informes y asesoramiento y formación. Además de la propuesta teórica se presentan diferentes ejemplos llevados a cabo en las Universidades de Navarra y Granada. El texto concluye subrayando la oportunidad laboral que supondría en el ámbito de la documentación este tipo de unidades.

Palabras Clave: universidad, unidad de bibliometría, sistema de información científica, indicadores bibliométricos, gestión I+D, apoyo a la investigación.

\section{Towards bibliometric units in universities: model and functions}

Abstract: In recent years, bibliometric indicators have been firmly established as a tool for research management and decision-making in the context of scientific policy. Spanish universities represent one sector that has seen significant growth in the use of indicators. These institutions now find themselves in an ever more competitive environment and therefore have a greater need to have knowledge of the performance of their researchers. Against this background, this paper presents a proposal for the creation of Bibliometric Units in Spanish universities and attempts specifically to illustrate both the model and functions of these units based on three pillars: control of information sources related to research, preparing reports, and advice/training. In addition to the theoretical proposal

\footnotetext{
* Grupo Evaluación de la Ciencia y la Comunicación Científica (EC3), Centro de Investigación Médica Aplicada, Universidad de Navarra.

** Grupo Evaluación de la Ciencia y la Comunicación Científica (EC3), Universidad de Granada. Correo-e: torressalinas@gmail.com.

Recibido: 16-03-2012; 2. ${ }^{\mathrm{a}}$ version: 18-04-2012; aceptado: 19-04-2012.
} 
we present different examples from the universities of Navarra and Granada. The text concludes that such units could represent an important opportunity for jobs in the field of Information Science.

Keywords: University, bibliometric unit, scientific information systems, bibliometric indicators, research management.

\section{La profesionalización de la bibliometría}

Los indicadores bibliométricos cobran un especial sentido cuando son empleados en el ámbito de la política científica contribuyendo al buen gobierno de la ciencia. Por remitirnos tan solo a nuestro contexto nacional en esta misma revista se publicó a finales de los ochenta el clásico discurso de Michael J. Moracsik (1989) "CCómo evaluar la ciencia y los científicos?". En el mismo se señalaban las claves sobre la utilidad la bibliometría para identificar a los agentes más capacitados de los sistemas científicos y como esta vertiente aplicada podía repercutir en la correcta reasignación de recursos o en el establecimiento de prioridades de investigación. Pero este uso de la Bibliometría no es nuevo y podemos rastrear sus orígenes en los pioneros trabajos de Narin de los años sesenta que dieron origen a la denominada como Bibliometría Evaluativa (Van Leeuwen, 2004). En mayor o menor grado esta función instrumental aparece subrayada en gran parte de la literatura posterior (Sancho, 1999; Braun, 1999; Camí, 2001; Askess, 2005). Frente a la bibliometría descriptiva la evaluativa se realiza en colaboración e interacción con el agente evaluado que ha de participar tanto al inicio del proceso, ofreciendo y facilitando la información necesaria, como al final, sopesando los indicadores resultantes para lo que es necesario en ocasiones contar con la opinión de expertos. Son, por tanto, necesarios dos elementos esenciales: la proactividad del evaluado y la precisión en los resultados lo que exige una gran exhaustividad en la recopilación de información y elaboración final de los indicadores.

Esta función asesora de la Bibliometría, a la que en Europa y en España han tratado de dar cobertura diferentes grupos e institutos como por ejemplo el CWTS (Universidad de Leiden, Holanda) o el antiguo ICYT (Consejo Superior de Investigaciones Científicas), ha sido habitual desde finales de los ochenta pero especialmente en los años noventa con el análisis del rendimiento de políticas nacionales y autonómicas (Maltrás y Quintanilla, 1995; Gómez y otros, 2007; Jiménez-Contreras y otros, 2011), programas de investigación (Espinosa de los Monteros y otros, 1999; Camí y otros, 2003) o centros de I+D y universidades (Torres-Salinas, 2007; Gómez y otros, 2010). Es precisamente el último ámbito de los señalados, el universitario, donde se ha producido una mayor penetración y aceptación. El entorno cada vez más competitivo en el que se mueven las universidades, marcadas sobre todo por la proliferación de rankings y la crisis económica, han puesto en evidencia ante los gestores de investigación la necesidad de contar con instrumentos que valoren el rendimiento científico de forma adecuada. Podemos decir que en el último lustro se ha ido creando un caldo de cultivo que ha faci- 
litado la aceptación de una "cultura de la evaluación científica» donde los indicadores bibliométricos se perciben como una pieza más en la toma de decisiones y el buen gobierno del sistema de educación superior. Este auge y asimilación se ha producido por la concurrencia de al menos tres factores:

1. La intensificación de los procesos de evaluación del profesorado y el personal de investigación. Desde que en 1989 la Comisión Nacional de Evaluación de la Actividad Investigadora (CNEAI) hiciera descansar sus criterios para la obtención de sexenios en indicios de calidad bibliométricos, sobre todo los asociados a las revistas científicas y al número de citas recibidas por las aportaciones (Ruíz-Pérez y otros, 2010), gran parte de los sistemas de evaluación posteriores como ANECA en su programa ACADEMIA han seguido este mismo modelo. De esta forma el profesorado ha acabado conociendo y aceptando, aunque no en todos los casos con el mismo nivel de acuerdo, los indicadores bibliométricos como uno de los ejes en la evaluación de sus carreras científicas.

2. La puesta en marcha por parte del extinto Ministerio de Ciencia e Innovación (MICINN) de diferentes programas como los Campus de Excelencia Internacional o las convocatorias Severo Ochoa. Dichos programas han establecido una nueva forma de financiación competitiva donde una buena parte del éxito de las convocatorias descansa en la presentación y seguimiento de indicadores muy relacionados con el rendimiento científico. Esto ha abocado a muchas universidades a la necesidad de contar con los instrumentos de medida necesarios.

3. La existencia de múltiples sistemas y plataformas para la evaluación de la investigación. Actualmente, debido precisamente a esta demanda, existe un número abundante de herramientas dedicadas a la evaluación de la investigación o susceptibles de serlo (Torres-Salinas y Cabezas-Clavijo, 2012; Cabezas-Clavijo y Torres-Salinas, 2012). Así junto a las índices tradicionales (Web of Science, Scopus, Google Scholar) encontramos suites tipo In-Cites, Scival y, además, proliferan los sistemas de información científica (SIC) que se perfilan como los candidatos idóneos para ser la fuente de información sobre la actividad científica de las universidades (Filippo y otros, 2011), pero cuyas posibilidades aún están por ser exploradas más profundamente.

Por tanto, por un lado las universidades se encuentran en la actualidad en un momento en el que se hace necesaria la introducción de políticas de evaluación asociadas a la investigación como pieza fundamental de su gobierno. Por el otro lado, estrechamente asociado con la puesta en funcionamiento de dichas políticas, se impone contar con fuentes de información sobre la actividad científica de los profesores lo suficientemente fiables, veraces y normalizadas para la posterior elaboración de indicadores bibliométricos a partir de ellas. Por ello, cabe preguntarse quién y cómo puede hacerse cargo de estas funciones. Pues bien, el objetivo de esta nota 
es proponer y narrar la experiencia de la creación de unidades de bibliometría en las universidades basada en la experiencia de los autores en diferentes instituciones como son los casos de las Universidades de Navarra y Granada donde se llevan a cabo experiencias de este tipo. Por ello, esencialmente en este trabajo nos centramos en describir el funcionamiento, tarea y procesos a desarrollar por estos nuevos servicios, así como presentar algunos ejemplos aplicados de los mismos.

\section{Propuesta de unidad de bibliometría}

Una unidad de bibliometría ha de entenderse como un servicio capaz de controlar todas las fuentes que almacenan los registros sobre cualquier tipo de actividad científica y tras diferentes procesos convertir dichas fuentes en registros útiles que puedan ser explotados ágilmente en la generación de conocimiento sobre las universidades. Por tanto, algunas de las competencias básicas están muy relacionadas con el conocimiento experto de fuentes de información, la creación de bases de datos y el posterior procesamiento, presentación y visualización de los indicadores que será lo que finalmente se consuma. Sobre esta premisa podemos decir que la unidad de bibliometría debe constituirse sobre tres ejes de trabajo o funciones fundamentales que describimos más detalladamente en los siguientes apartados (tabla 1 ):

1. Control de las fuentes de información sobre investigación.

2. Generación de informes de análisis, prospectiva y vigilancia.

3. Formación, asesoramiento y consulta experta.

TABLA 1

Los pilares básicos y las funciones de una unidad de bibliometría

\begin{tabular}{|c|c|c|}
\hline 1 & 2 & 3 \\
\hline $\begin{array}{l}\text { Control de las fuentes } \\
\text { información } \\
\text { sobre investigación }\end{array}$ & $\begin{array}{c}\text { Generación de informes } \\
\text { de análisis, prospectiva } \\
\text { y vigilancia }\end{array}$ & $\begin{array}{c}\text { Formación, } \\
\text { asesoramiento } \\
\text { y consulta experta }\end{array}$ \\
\hline $\begin{array}{l}\text { - Identificación. } \\
\text { - Control. } \\
\text { - Normalización. } \\
\text { - Unificación. }\end{array}$ & $\begin{array}{l}\text { - Memorias de investigación. } \\
\text { - Informes estratégicos. } \\
\text { - Informes de divulgación. } \\
\text { - Informes convocatorias. }\end{array}$ & \multirow[t]{2}{*}{$\begin{array}{l}\text { Asesoramiento personalizado } \\
\text { al profesorado para la prepa- } \\
\text { ración de solicitudes a agen- } \\
\text { cias como CNEAI o ANECA. }\end{array}$} \\
\hline$\downarrow$ & $\downarrow$ & \\
\hline $\begin{array}{l}\text { Creación de sistema informáti- } \\
\text { co para cálculo de indicadores. }\end{array}$ & $\begin{array}{l}\text { Divulgación de resultados } \\
\text { dentro y fuera universidad. }\end{array}$ & \multirow{2}{*}{$\begin{array}{l}\text { Formación mediante cursos } \\
\text { con el objetivo de mejorar los } \\
\text { resultados científicos de la } \\
\text { universidad. }\end{array}$} \\
\hline $\begin{array}{l}\text { - Consultas precalculadas. } \\
\text { - Cuadros de mando. } \\
\text { - Exportación a otros sistemas. }\end{array}$ & $\begin{array}{l}\text { - Gabinetes de prensa. } \\
\text { - Prensa local. } \\
\text { - Redes Sociales. }\end{array}$ & \\
\hline
\end{tabular}




\subsection{Control de las fuentes de información sobre investigación}

Es ampliamente conocido que uno de los aspectos fundamentales para el desarrollo de cualquier servicio/trabajo bibliométrico descansa necesariamente en la identificación, control, normalización y unificación de todas las fuentes de información susceptibles de contener datos sobre la actividad científica de una institución. La construcción de registros integrados es esencial ya que el buen funcionamiento y rendimiento de los procesos posteriores depende en buena medida de la información con la que contemos. Dentro de este proceso podemos distinguir dos tipologías de fuentes que se han de tener en cuenta. Las primeras de éstas son los tradicionales índices de citas como son Web of Science y los productos que nos ofrecen indicios de calidad como pueden ser el Journal Citation Reports (JCR). Por otro lado estarían las bases de datos internas de la propia universidad que suelen estar dispersas por diversos servicios, como suele ocurrir con las de contratos, proyectos de investigación, tesis doctorales o datos más singulares y sometidos a algún tipo de protección legal como el caso de los sexenios del profesorado. Actualmente esta fase de control de la actividad científica se puede reducir considerablemente si las universidades cuentan con sistemas de información científica basados en el estándar Currículo Vitae Normalizado (CVN) (Báez y otros, 2008) ya que éstos compilan los currícula de los investigadores de una institución, aunque no siempre de forma fiable y exhaustiva.

Por tanto, es necesario tener un conocimiento preciso de la información de la que disponemos ya que cada una de ellas por separado solo ofrece un retrato parcial, por lo que hay que recurrir a la unificación o al menos a la combinación de la mismas. Por ejemplo, un SIC puede ofrecer una imagen muy detallada de la actividad científica de una universidad; sin embargo, para obtener indicadores basados en el Impact Factor es necesario cruzar esta información con los ficheros normalizados del JCR. Por tanto, la unidad ha de estar en condiciones no solo de controlar las fuentes sino de combinarlas entre sí para la posterior generación de indicadores. Este proceso de unificación en una o varias bases de datos implica, además, procesos de normalización (gestión de nombres de los profesores, eliminación de registros duplicados, creación de clasificaciones científicas, etc.) que garantice la calidad y posterior uso de la información. Toda esta labor ha de ser, además, periódica, con una actualización y mantenimiento constante, de forma que permita a la unidad disponer de los datos para su consulta en cualquier momento. Sin duda, una vez que conseguimos un registro bibiométrico único éste puede complementarse con una colección de consultas predefinidas y habituales que nos facilitan obtener los indicadores con rapidez para cualquier agente de nuestros sistemas; en última instancia el objetivo sería que esas consultas alimentaran cuadros de mando.

Un ejemplo de este tipo de sistemas (figura 1) es el desarrollado en la Universidad de Navarra con el nombre de "Científica" (Torres-Salinas, 2007) y que se especializa en este caso en el control de la producción científica indexada en Web of Science y Scopus, incorpora, además, los JCR completos y combina dichos 
productos con las bases de datos de centros, departamentos e investigadores. Una de sus funciones principales es la generación final de cuadros de mandos bibiométricos de producción e impacto de diferente naturaleza ( $\mathrm{n}^{\circ}$ Documentos Citables, n. ${ }^{\circ}$ Documentos en Primer Cuartil, Factor de Impacto Normalizado, n. ${ }^{\circ}$ de Citas, etc.) de los diferentes agentes de esta universidad (investigadores y departamentos). En la Universidad de Granada la solución adoptada es similar, pero en este caso la aplicación integra otro tipo de fuentes como es el caso de los registros del profesorado, bases de datos de proyectos de investigación y contratos OTRI, los JCR, la Clasificación Integrada de Revistas Científicas (CIRC) y los datos normalizados provenientes del Sistema de Información Científica de Andalucía (SICA2). El sistema se actualiza periódicamente y al igual que el anterior permite obtener cuadros de mando y consultas directas en pantalla.

\section{FIGURA 1}

Cuadro de Mando automatizado para investigadores y departamentos del sistema de información "Científica" de la Universidad de Navarra

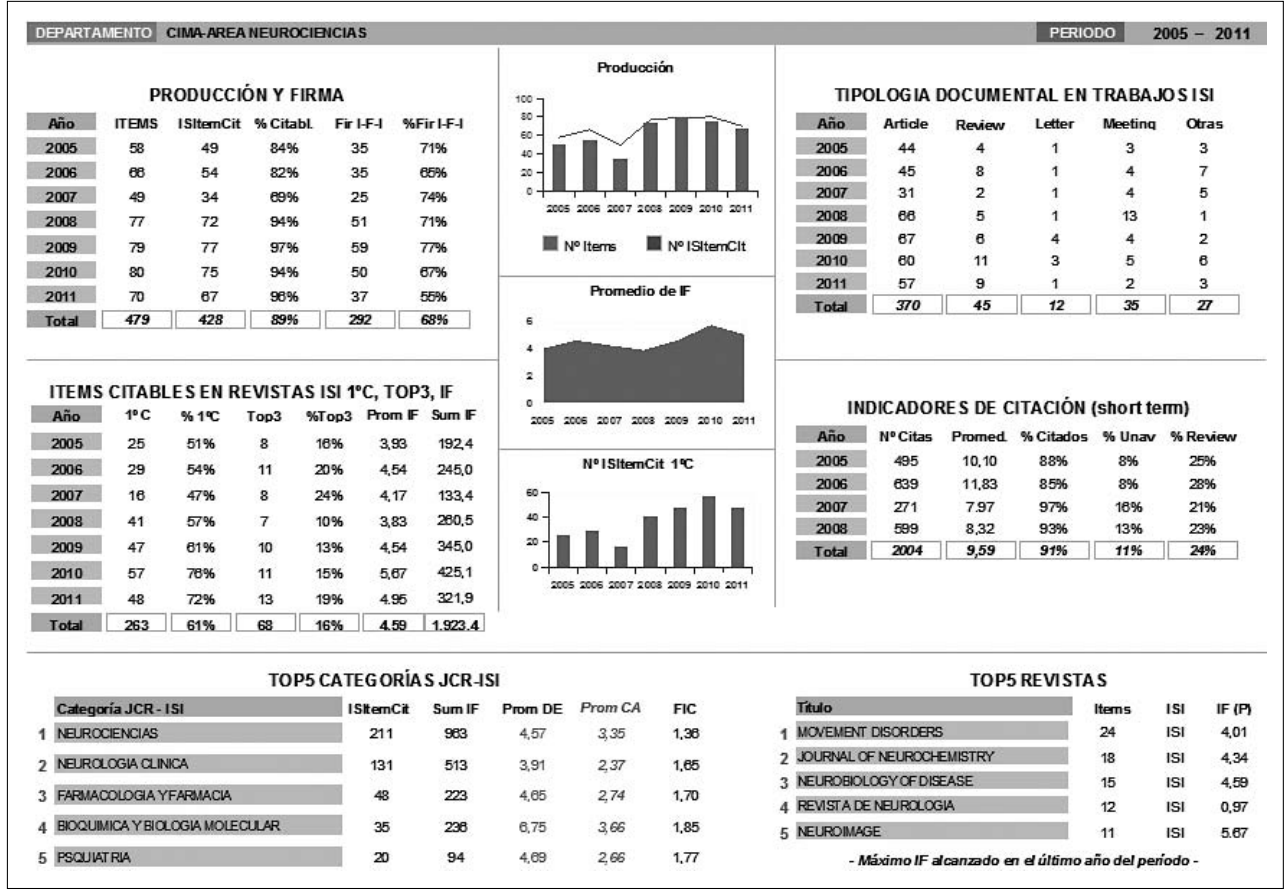

\subsection{Generación de informes de análisis, prospectiva y vigilancia}

Una vez que se controlan las fuentes de información el siguiente objetivo ha de pasar necesariamente por la generación de conocimiento sobre los sistemas 
de $\mathrm{I}+\mathrm{D}$ de las universidades y sus resultados. Dichos informes deben atender a dos tipos de demandas fundamentales. En primer lugar, aquéllas que se producen con frecuencia por parte de las diferentes unidades de la universidades (departamentos, institutos, centros de investigación, etc.) y que se pueden solventar con rapidez, como hemos comentado anteriormente, con la automatización en consultas y cuadros de mando estandarizados. En segundo lugar estarían aquellos relacionados con los planes estratégicos de las universidades y que tienen como objetivo un mejor conocimiento de las fortalezas y debilidades del sistema I+D, por tanto, tienen un carácter más exhaustivo y orientado a los objetivos de la institución y su verificación. Estos informes no solo deben estar focalizados en la exposición de los resultados de investigación a través de indicadores bibliométricos sino que también ha de ponerse un especial énfasis en la contextualización de los mismos, por lo que en ocasiones son necesarios indicadores más complejos y comparaciones con otras universidades (benchmarking). En este contexto algunos de los informes que se pueden realizar, y de hecho se vienen realizando de forma habitual en las mencionadas universidades, dentro de las unidades de bibliometría son los siguientes:

- Informes bibliométricos "convencionales" de carácter anual sobre el estado de la investigación para su incorporación como memorias de investigación.

- Informes bibliométricos especializados en un aspecto concreto de la investigación de la universidad que tenga un interés estratégico para la universidad.

- Informes bibliométricos de divulgación de aspectos destacados de la investigación y dirigidos a un público más amplio y no experto.

- Informes para convocatorias que supongan una fuente de financiación a la universidad: CEI, Innocampus o Severo Ochoa u otros sellos de calidad.

El último eslabón dentro de este bloque de trabajo es la divulgación de los resultados de los diferentes informes en un contexto diferente al de la propia gestión. La información bibliométrica puede tener un gran interés social y puede ser un aliado interesante a la hora promocionar nuestras instituciones. En este sentido es importante el trabajo conjunto que se puede realizar con los gabinetes de comunicación de las universidades, sobre todo mediante la redacción de notas de prensa que pueden llegar a los diferentes medios de comunicación. Igualmente también pueden ser de interés la divulgación que se obtiene a través de la creación de perfiles en redes sociales tipo Twitter o Facebook pudiéndose emplear un ciclo de difusión similar al de otro tipo de materiales (Torres-Salinas y Delgado, 2009). Esta divulgación de resultados no solo debería limitarse a los resultados generados por la unidad, sino que pueden tratarse otros aspectos como la reseña de diferentes logros científicos (premios, trabajos más citados, financiaciones importantes, publicaciones en revistas top).

En este sentido por ejemplo, tanto en la Universidad de Granada como en la de Navarra se publican informes periódicos a modo de memoria de investigación. Concretamente en la Universidad de Navarra el último publicado es «Diez Años de 
investigación en la universidad de Navarra. 2001-2011" y en la Universidad de Granada la "Memoria Básica de Investigación 2011" (VPCI-UGR, 2011a). En el caso de los informes monográficos que subrayan aspectos concretos podemos mencionar: "Mujer e Investigación en la UGR" (VPCI-UGR, 2011b) o "La Universidad de Granada en los rankings ISI" (VPCI-UGR, 2011c). Por último, para acercar los indicadores bibliométricos a un público más amplio hay que señalar la creación en la UGR de un boletín trimestral de dos páginas denominado "Hoja Bibliométrica" de la que actualmente se han publicado tres números (figura 2) (VPCI-UGR, 2011d, 2011e, 2012). En el plano de divulgación en la UGR, gracias al envío de notas de prensa resumiendo dichos informes, se ha conseguido que algunos de ellos sean mencionados por la prensa local. En general el objetivo último de esta divulgación es intentar salvar y reducir el divorcio o la ignorancia tradicional del ciudadano sobre las actividades de la institución universitaria en materia de investigación.

\section{FIGURA 2}

Portada de una de las "Hojas Bibliométricas" publicadas en la Universidad de Granada para divulgar los resultados de investigación

\begin{tabular}{|c|c|c|c|c|c|}
\hline $\begin{array}{c}\text { HDJA } \\
\text { BIBLIDMÉTRICA } \\
\text { Universidad de Granada }\end{array}$ & TIE E & ] & A INVESTIE & & \multirow[t]{2}{*}{$\aleph_{\text {http://citic.ugr.es }}$} \\
\hline \multirow{2}{*}{$\# 01$ Jukio } & \multicolumn{4}{|c|}{$\begin{array}{l}\text { Producción de las universidades españolas en TIC } \\
\qquad 2006-2010\end{array}$} & \\
\hline & Número de documento & & \multicolumn{2}{|c|}{ Número de Citas } & \multirow{11}{*}{$\begin{array}{l}\text { ALGUNAS DE LAS PRINCIPALES } \\
\text { LINEAS DE INVESTIGACIÓN DEL CITIC } \\
\text { Nanoelectronics, Computer Vision, Image } \\
\text { Recognition, Soft Computing (including } \\
\text { fuzzy logic, metaheuristics and neural } \\
\text { networks), Data Mining, Decision Support } \\
\text { Systems, Computational Biology, } \\
\text { Bioinformatics, Mobile and Sensor } \\
\text { Networks, Optimization, Planning. }\end{array}$} \\
\hline \multirow{5}{*}{$\begin{array}{l}\text { \#EI centro de investigación } \\
\text { CITIC-UGR se creó en el } \\
\text { año } 2007 \text { y aglutina a los } \\
\text { investigadores sobre TIC. }\end{array}$} & Pditécnica Cataluña & 859 & Granada & 2205 & \\
\hline & Politécnica Valencia & 598 & Pditécnica C ataluña & 2183 & \\
\hline & Pofitécnica Madid & 517 & Politécnica Valencia & 1690 & \\
\hline & Granada & 502 & Politécrica Machid & 1312 & \\
\hline & Carlos III I & 412 & Carlos III [ & 971 & \\
\hline \multirow{6}{*}{$\begin{array}{l}\text { \# En la actualidad está } \\
\text { integrado por } 23 \text { grupos de } \\
\text { investigación. } \\
\text { \#EI CITIC-UGR se ha } \\
\text { presentado a la } \\
\text { convocatoria de Centros de } \\
\text { Excelencia Severo Ochoa. }\end{array}$} & \multicolumn{2}{|l|}{ Índice $\mathrm{H}$} & \multicolumn{2}{|c|}{$\%$ de documentos en el $1^{\circ} \mathrm{C}$} & \\
\hline & Granada & 20 & Granada & $34 \%$ & \\
\hline & Politécnica Valencia & 16 & Poitécrica Mactid & $32 \%$ & \\
\hline & Politécnica Cataluña & 14 & Pditécnica C ataluña & $30 \%$ & \\
\hline & Politécnica Mactid] & 13 & Carlos III & $30 \%$ & \\
\hline & Carios III I & 13 & Politécnica Valencia [ & $24 \%$ & \\
\hline \multirow{5}{*}{$\begin{array}{l}\text { \# Las TIC son uno de los } \\
\text { pilares del Campus de } \\
\text { Excelencia Internacional } \\
\text { BioTic. }\end{array}$} & \multicolumn{2}{|l|}{ Promedio de Citas } & \multicolumn{2}{|c|}{$\%$ documentos muy citados } & \\
\hline & Granada & 4,39 & Granada & $19 \%$ & \\
\hline & Politécnica Valencia & 2,82 & Politécnica Valencia & $11 \%$ & \\
\hline & Pditécnica Cataluña & 2,54 & Poditécnica Cataluña & $9 \%$ & \\
\hline & Pofitécnica Mactid & 2,53 & Politécrica Mactid & $9 \%$ & $\circ$. \\
\hline Vicerrectorado de Politica & Carlos III I & 2,35 & Carlos III I & $8 \%$ & $2+1121$ \\
\hline $\begin{array}{c}\text { Cientifica e Investigación } \\
\text { mugrinvesiga }\end{array}$ & & & & & $\begin{array}{l}\text { Fotos: Muriel Romero Sánchez } \\
\text { http: } / / \text { www.etringita.com }\end{array}$ \\
\hline
\end{tabular}

\subsection{Formación, asesoramiento y consulta experta}

El tercer pilar sobre el que debería descansar una unidad de bibliometría es aprovechar los conocimientos de sus responsables para el asesoramiento y la 
consulta experta. Esta parte del servicio incluiría diferentes tipos de consulta. En primer lugar el asesoramiento individualizado a los profesores sobre los procesos de evaluación de las agencias de evaluación (CNEAI y ANECA) así como asesoramiento en la preparación de recursos y alegaciones cuando se produzcan evaluaciones negativas en dichas convocatorias. Por otro lado, también se puede ofrecer este servicio a través de cursos realizados en las propias facultades, programas de doctorado, etc. Este tipo de cursos, además de los mencionados de las agencias, se pueden complementar con otros de temática diferente como el manejo de bases de datos y productos dedicados a la evaluación científica, así como en prácticas de publicación científica o difusión de resultados. En definitiva, se trata de que mediante esta parte del servicio se aumente la tasa de éxito en las solicitudes de las agencias y por otro, de intentar mejorar los resultados de investigación en términos de publicación mediante la formación en buenas prácticas de comunicación científica.

Un ejemplo concreto de este tipo de asesoramiento y formación del profesorado y personal investigador es la puesta en marcha en la UGR de la denominada "Aula de Formación". En ella se ofrece formación a grupos reducidos de la comunidad investigadora de la UGR. Éstos pueden solicitar cursos individuales o bien formar módulos integrando diferentes cursos o bien incluirlos, por ejemplo, en jornadas de formación de jóvenes investigadores o programas de doctorado. Desde que se puso en marcha el "Aula de Formación" en noviembre de 2011 se han impartido más de 12 sesiones por diferentes facultades y centros, que han sumado unos 600 asistentes en total. Algunos de los cursos que más atención han atraído han sido los titulados "Talleres de preparación de solicitud de sexenios» o "Cómo escribir y publicar en revistas científicas de Impacto». A título informativo, a continuación se ofrece la relación de cursos que se ofertan en la actualidad:

- Cómo utilizar los indicadores bibliométricos para la obtención de sexenios.

- Cómo utilizar los indicadores bibliométricos para la acreditación de ANECA.

- Técnicas e indicadores bibliométricos para la evaluación de la Actividad Científica.

- Cómo comunicar y diseminar tus resultados científicos a través de la web.

- Cómo publicar en revistas científicas de Impacto.

- Cómo preparar la solicitud de un proyecto de investigación competitivo.

- Búsquedas en bases de datos científicas: del rescate de citas a la creación de perfiles.

- Cómo gestionar un proyecto de investigación.

\section{A modo de conclusión}

En estas páginas se ha presentado una propuesta y ejemplo de lo que entendemos que debería ser un modelo básico de Unidad de Bibliometría en un contexto universitario y como prestar este servicio de un modo centralizado para 
obtener el mejor rendimiento. Si bien es cierto que parcialmente algunas de las funciones señaladas se vienen prestando por otros servicios de las universidades, como puede ser el caso de las bibliotecas universitarias u OTRIS, desde aquí abogamos por crear unidades con una cobertura más amplia y con mayor visibilidad dentro de los organigramas de las universidades. Idealmente una Unidad de Bibliometría debería depender de los vicerrectorados de investigación ya que éstos, por un lado, son los que generan la mayor parte de las fuentes que recopilan los datos sobre la actividad investigadora del profesorado y, por otro lado, son los destinatarios directos y máximos interesados en la mayor parte de la información y conocimiento que se genere desde la unidad. En cualquier caso el lugar que se ocupe dentro del organigrama depende de las propias universidades, pero es evidente que un factor a tener en cuenta son las actividades bibliométricas desarrolladas previamente a la creación de la unidad por vicerrectorados, bibliotecas y OTRIS, situación que obliga a una necesaria coordinación, planificación y reparto de competencias antes de la creación de la unidad. Asimismo este tipo de servicios requiere para su buen funcionamiento y autonomía de un personal multidisciplinar que abarque no solo conocimientos sobre bibliometría y evaluación de la actividad científica, si no también otra formación como técnicas estadísticas, visualización de la información, informática (programación y bases de datos) o divulgación científica. Otro aspecto reseñable es el paso de la bibliometría evaluativa de los grupos de investigación a una fase de mayor profesionalización de la práctica bibliométrica. La documentación, desde un punto de vista laboral, debe abrirse a nuevos nichos de trabajo y ésta que hemos comentado en estas páginas creemos que es una gran oportunidad.

\section{Bibliografía}

Aksnes, D. W. (2005): Citation and their use as indicators in science policy. Studies of validity and applicability issues with a particular focus on highly cited papers. University of Twente, Twente (Tesis doctoral).

Báez, J. M.; Peset, F.; Núñez, F.; Ferrer-Sapena, A. (2008): CVN: normalización de los currículos científicos. El Profesional de la Información, vol. 17 (2), 212-220

Braun, T. (1999): Bibliometric indicators for the evaluation of universities: intelligence from the quantization of the scientific literature. Scientometrics, vol. 45 (3), 425-32.

Cabezas-Clavijo, A., y Torres-Salinas, D. (2012): Google Scholar Citations y la emergencia de nuevos actores en la evaluación de la investigación. Anuario Thinkepi, vol. 6, pp. $147-153$.

Camí, J. (2001): Evaluación de la investigación biomédica. Medicina Clínica (117), 510-513.

Camí, J.; Suñen, E.; Carbó, J., y Coma, L. (2003): Producción científica española en Biomedicina y Ciencias de la Salud (1994-2000). Informe del Instituto de Salud Carlos III - Fondo de Investigación sanitaria. Quark (30), 13-79.

Espinosa de los Monteros, J.; Díaz, V.; Toribio, M. A. y otros (1999): La investigación biomédica en España (I). Evaluación del Fondo de Investigación Sanitaria (FIS) a través 
de los proyectos de investigación financiados en el período 1988-1995 a instituciones sanitaria asistenciales (hospitales). Medicina Clínica (112), 182-97.

Filippo, D.; Sanz-Casado, E.; Urbano Salido, C.; Ardanuy, J., y Gómez-Caridad, I. (2011): El papel de las bases de datos institucionales en el análisis de la actividad científica de las universidades. Revista Española de Documentación Científica, vol. 34 (2), 165-189.

Gómez, I.; Fernández, M. T.; Bordons, M., y Morillo, F. (2007): Indicadores de producción científica y tecnológica de la Comunidad de Madrid. Dirección General de Universidades e Investigación de la CM, Madrid.

Gómez, I.; Bordons, M.; Morillo, F.; Moreno, L.; Aparicio, J.; Diaz-Faes, A. A., y GonzálezAlbo, B. (2011): La actividad científica del CSIC a través de indicadores bibliométricos (web of Science 2006-2010). Madrid: IEDCYT-CCHS-CSIC. En: http://hdl.handle.net/ $10261 / 48118$.

Jiménez-Contreras, E.; Cabezas-Clavijo, A.; Delgado-López-Cózar, E.; Ruiz-Pérez, R., y Tomás López, M. (2011): Análisis de la producción y actividad científica de la Región de Murcia 1999-2009. Murcia: Fundación Séneca.

Maltrás, B., y Quintanilla, M. A. (1995): Indicadores de la producción científica: España, 1986-91. Madrid: Consejo Superior de Investigaciones Científicas.

Moravcsik, M. J. (1989): ¿Cómo evaluar la ciencia y a los científicos? Revista Española de Documentación Científica, vol. 12 (3), 313-25.

Ruiz-Pérez, R.; Delgado López-Cózar, E., y Jiménez Contreras, E. (2010): Principios y criterios utilizados en España por la Comisión Nacional Evaluadora de la Actividad Investigadora (CNEAI) para la valoración de las publicaciones científicas: 1989-2009. Psicothema, vol. 22 (4), 898-908.

Torres Salinas, D. (2007): Diseño de un sistema de información y evaluación científica. Análisis ciencimétrico de la actividad investigadora de la Universidad de Navarra en el área de ciencias de la salud (1999-2005). Granada: Universidad de Granada.

Torres-Salinas, D., y Delgado López-Cózar, E. (2009). Estrategia para mejorar la difusión de los resultados de investigación con la Web 2.0. El Profesional de la Información, vol. 19 (5), 534-539.

Torres Salinas, D.; Cabezas-Clavijo, A. (2012): Herramientas para la evaluación de la ciencia en universidades y centros I+D: descripción y usos. Anuario Thinkepi, vol. 6, pp. 142-146.

Sancho, R. (1999): Indicadores bibliométricos utilizados en la evaluación de la ciencia y la tecnología. Revisión bibliográfica. Revista Española de Documentación Científica, vol. 13 (3-4), 842-865.

Van Leeuwen, T. (2004): Descriptive versus evaluative bibliometrics. En H. Moed; W. Glänzel, y U. Schmorch (ed.). Handbook of quantitative science and technology research: the use of publication and patents statistics in studies of SET systems. Kluwer, Dordecht.

VPCI-UGR - Vicerrectorado de Política Científica e Investigación (2011a): Memoria Básica de Investigación 2011. http://investigacion.ugr.es/ugrinvestiga/pages/doc_ugrcifras/ memoriainvestigacion2011_versionugrinvestiga/! [10/03/2012].

VPCI-UGR - Vicerrectorado de Política Científica e Investigación (2011b): Mujer e Investigación en la UGR. http://investigacion.ugr.es/ugrinvestiga/pages/doc_ugrcifras/informemujereinvestigacionugr/! [10/03/2012]. 
VPCI-UGR - Vicerrectorado de Política Científica e Investigación (2011c): La Universidad de Granada en los rankings ISI. http://investigacion.ugr.es/ugrinvestiga/pages/doc_ugrcifras/launiversidaddegranadaenlosrankingsisi/! [10/03/2012].

VPCI-UGR - Vicerrectorado de Política Científica e Investigación (2011d): Hoja Bibliométrica 1. TIC en la UGR: la investigación en el CITIC UGR. http://investigacion.ugr.es/ ugrinvestiga/pages/doc_ugrcifras/hojabibliometrica1_junioticenlauniversidaddegrana$\mathrm{da} / !$ [10/03/2012].

VPCI-UGR - Vicerrectorado de Política Científica e Investigación (2011e): Hoja Bibliométrica 2. Los Citation Classics de la Universidad de Granada. http://investigacion.ugr. es/ugrinvestiga/pages/doc_ugrcifras/hojabibliometrica2_octubrecitationclassics/! [10/03/2012].

VPCI-UGR - Vicerrectorado de Política Científica e Investigación (2011e): Hoja Bibliométrica 3. La colaboración científica internacional de la Universidad de Granada. http:// investigacion.ugr.es/ugrinvestiga/pages/doc_ugrcifras/hojabibliometrica3_marzo_colaboracioninternacional/! [15/04/2012]. 